\title{
Retrospective case series of clinical outcomes associated with chiropractic management for veterans with low back pain
}

\author{
Andrew S. Dunn, DC, MS, MEd; ${ }^{1-2 *}$ Bart N. Green, DC, MSEd; ${ }^{3}$ Lance R. Formolo, DC, MS; ${ }^{1-2}$ David \\ Chicoine, BS $^{2}$ \\ ${ }^{1}$ Department of Veterans Affairs (VA) Western New York Healthcare System, Buffalo VA Medical Center, Buffalo, NY; \\ ${ }^{2}$ New York Chiropractic College, Seneca Falls, NY; ${ }^{3}$ Department of Physical and Occupational Therapy, Chiropractic \\ Division, Naval Medical Center San Diego, Marine Corps Air Station, Miramar Branch Medical Clinic, San Diego, \\ CA, and National University of Health Sciences, Lombard, IL
}

\begin{abstract}
Musculoskeletal complaints, most notably low back pain (LBP), are prevalent among veterans. Despite a focus on LBP management by chiropractors within the Veterans Health Administration, limited published accounts detail clinical outcomes with chiropractic management of LBP among veterans. This was a retrospective case series of 171 veterans with a chief complaint of LBP who were managed with chiropractic care. Descriptive statistics and paired $t$-tests were used, with the Numeric Rating Scale (NRS) and the Back Bournemouth Questionnaire (BBQ) serving as the outcome measures. A minimum clinically important difference (MCID) was set as $30 \%$ improvement from baseline for both measures. The mean number of treatments was 8.7. For the NRS, the mean raw score improvement was 2.2 points, representing $37.4 \%$ change from baseline; 103 (60.2\%) patients met or exceeded the MCID. For the BBQ, the mean raw score improvement was 13.6 points, representing $34.6 \%$ change from baseline; 92 patients (53.8\%) met or exceeded the MCID. For this sample of veterans with LBP, the mean percentages of clinical improvement were statistically significant and clinically meaningful for both the NRS and BBQ.
\end{abstract}

Key words: chiropractic, hospitals, low back pain, musculoskeletal and neural physiological phenomena, Operation Enduring Freedom, Operation Iraqi Freedom, outcome assessment, rehabilitation, spinal manipulation, veterans.

\section{INTRODUCTION}

Low back pain (LBP) is a costly and common chronic condition among veterans receiving care within the Veterans Health Administration (VHA) [1-2]. Between 2000 and 2007, the annualized percentage rate increase in the number of veterans with LBP receiving care within VHA was 4.8 percent per year [2]. This exceeded the rate increases for depression, diabetes, and hypertension [2]. This increasing prevalence of LBP is evident in reports among recently returning veterans [3-4]. A survey of 15,000 Persian Gulf war veterans revealed a prevalence of

Abbreviations: $\mathrm{BBQ}=$ Back Bournemouth Questionnaire, $\mathrm{CI}=$ confidence interval, $\mathrm{FD}=$ flexion distraction, $\mathrm{OIF} / \mathrm{OEF}=$ Operation Iraqi Freedom/Operation Enduring Freedom, LBP = low back pain, MCID = minimum clinically important difference, NRS = Numeric Rating Scale, PTSD = posttraumatic stress disorder, SMT = spinal manipulative therapy, VA = Department of Veterans Affairs, VAWNYHS = VA Western New York Healthcare System, VHA = Veterans Health Administration.

* Address all correspondence to Andrew S. Dunn, DC, MS, MEd; VA Western New York Healthcare System, Buffalo VA Medical Center, 3495 Bailey Avenue, Buffalo, NY 14215; 716-862-8543; fax: 716-862-7248.

Email: andrew.dunn@va.gov

DOI:10.1682/JRRD.2010.10.0202 
back pain of 45 percent [3]. Among a sample of Operation Iraqi Freedom/Operation Enduring Freedom (OIF/ OEF) veterans with chronic pain, the back (46.4\%) was the most frequent site of pain [4].

Chiropractic services were recently added to VHA [5], and components of chiropractic services have been shown to be of benefit for LBP in the general population [6-8]. Data are starting to emerge on chiropractic services for veteran populations [9-11]. The majority of the chiropractors providing care within VHA identified LBP as the most common presenting complaint among veteran patients [12]. LBP and chiropractic care have been touched upon only briefly in previous veteran studies, including one case report [9], a case series looking at processes and outcomes of care for OIF/OEF veterans at one clinic [10], and a retrospective study that focused on the potential influence of posttraumatic stress disorder (PTSD) on outcomes of care in veterans with neck or back pain [11]. However, no publications to date have focused solely on chiropractic treatment outcomes for LBP in the veteran population.

The specific aim of this study was to report demographic characteristics, chiropractic treatment methods and frequency, and clinical outcomes for chiropractic management of LBP in a sample of veteran patients. The hypothesis was that statistically significant improvements in LBP would be realized following treatment for this sample of veterans.

\section{METHODS}

\section{Design}

This study was a retrospective case series of a prospectively maintained quality assurance data set. The study proposal was reviewed and approved through the Department of Veterans Affairs (VA) Western New York Healthcare System (VAWNYHS) Research and Development Committee and Institutional Review Board.

\section{Sample}

The chiropractic clinic at VAWNYHS was the setting for this study; it serves the veteran population in the western New York region. Veterans access chiropractic services through gatekeeper consultation requests, generally through primary care within the medical center or surrounding VA community-based outpatient health centers. In the calendar year between January 1, 2009, and
December 31, 2009, treatment was initiated for 253 out of the 289 veterans with a presenting complaint of LBP and chiropractic consultations completed within the clinic. Clinical outcomes representing change scores from baseline to discharge were included for analysis for patients with completed baseline and discharge outcome measures, including a Numeric Rating Scale (NRS) and the Back Bournemouth Questionnaire (BBQ), and a minimum of two chiropractic treatments.

\section{Chiropractic Treatment Methods and Frequency}

The number of treatments for each patient was determined by frequency count. The typical course of care consisted of one to two treatments a week, with a reevaluation and review of updated outcome measures after every fourth treatment or earlier as indicated. The type of treatment provided was noted per case, with many treatment encounters incorporating more than one form of manual treatment. Treatments were provided by two chiropractors along with contributions from supervised chiropractic students. Manual treatments for the low back region, alone or in combination, included flexion distraction (FD), spinal manipulative therapy (SMT), and spinal mobilization. FD is a manual procedure designed to produce continuous passive spinal motion in a prone posture with elements of traction assisted by manual pressure applied to the low back region [13]. SMT is a form of manually assisted passive motion involving a highvelocity, low-amplitude thrust to the spine that is generally applied with the patient positioned in a lateral decubitus posture [13]. Spinal mobilization involves repetitive joint oscillations without the high-velocity, low-amplitude thrust associated with SMT [13]. Patients also received instructions for therapeutic exercises and stretches tailored to the nature of their presentation.

\section{Clinical Outcomes}

Clinical outcome measures were pain severity (NRS) and a biopsychosocial measure of LBP symptoms (BBQ). The NRS within this study was obtained as a verbal rating of pain severity on a scale of 0 to10 at the time of care [14]. The BBQ is a multidimensional outcome measure based on the biopsychosocial model covering the pain, disability, affective, and cognitive-behavioral dimensions of musculoskeletal conditions [15-17]. The $\mathrm{BBQ}$ is a 7-question instrument with scores ranging from 0 to 70 , with higher scores representing increased symptom severity. Versions of the Bournemouth Questionnaire 
have been validated for both neck and back complaints [15-17]. In addition to pain severity and the effect of pain on social, work, and other daily activities, the questionnaire also provides measures of depression and anxiety. Both the NRS and BBQ were obtained as part of clinical practice by the treating chiropractors at baseline and at the time of presentation after four treatments or sooner if indicated. The NRS and BBQ were used as elements of case management to help identify whether or not patients were responding to treatments and guide clinical decision-making with regard to continuing to provide care or moving to discharge if subjects were not responding or reaching a plateau in terms of clinical progress. Reevaluation after every four treatments or sooner was established based upon local practice parameters to identify clinical end points as early as reasonably possible and to best ensure appropriate access to care, while balancing the benefit to each veteran with the allocation of relatively limited resources.

A published account for the BBQ found a raw score change of $\geq 14$ points or a percentage change of $\geq 47$ percent to be clinically significant improvement [18]. However, the mean baseline BBQ in that study was 29.8, which was lower than the current study, with considerable demographic differences in terms of age, sex, and chronic versus acute LBP compared with the current study [18]. As percentage change scores can account for variations in baseline values and can be interpreted across outcomes measures, a percentage change score was used over a raw change score for the outcome measures employed. Based upon published accounts of an international consensus for a range of commonly used back pain outcome measures [19], the minimum clinically important difference (MCID) was considered as a 30 percent change from baseline for both the NRS and BBQ for the current study. The percentage of patients who reached or exceeded the MCID for the measures used was reported.

\section{Data Analysis}

The independent variable was chiropractic management consisting of a pragmatic treatment approach including, alone or in combination, FD, SMT, and spinal mobilization. The dependent variables were NRS and BBQ scores. Descriptive statistics, including mean, standard deviation, and 95 percent confidence interval (CI), for continuous variables and proportions for categorical variables were used to describe the demographic characteristics of the sample. Match paired $t$-tests were used in a two-tailed fashion to evaluate clinical outcomes in terms of change from baseline to discharge for both the NRS and BBQ $(p<0.05)$. Analyses were conducted using JMP version 5.1 (SAS Institute, Inc; Cary, North Carolina).

\section{RESULTS}

\section{Demographics}

The Table presents the detailed demographic data. Out of the 253 consults that resulted in the initiation of a course of care, analysis was carried out for the 171 veterans (67.6\%) who met the inclusion criteria with completed baseline and discharge outcome measures and a minimum of two treatments. The average patient was an obese, 53-year-old male with chronic LBP. The most prominent period of military service represented was the Vietnam era (40.4\%) followed by OIF/OEF (21.6\%). Many of the patients in the sample had various levels of service-connected disability, meaning they were “. . . disabled by an injury or illness incurred or aggravated during active military service" [20]. Identified from diagnoses embedded within the clinical record, we reported the number of veteran patients presenting with complicating spinal conditions to further represent the complexity of this sample. Seven patients (4.1\%) had previously undergone lumbar surgery, and eight patients (4.7\%) had lumbar spondylolysis.

\section{Chiropractic Treatment Methods and Frequency}

A flowchart of the course of care is presented in Figure 1. The mean number of treatments per case was

Table.

Demographic characteristics of veterans with LBP $(n=171)$.

\begin{tabular}{lc}
\hline \multicolumn{1}{c}{ Characteristic } & $\boldsymbol{n}(\%)$ or Mean (95\% CI) \\
\hline Sex (Male) & $158(92.4)$ \\
Age (yr) & $53.3(50.8-55.9)$ \\
BMI (kg/m $\left.{ }^{2}\right)$ & $30.1(29.2-31.0)$ \\
Chronic LBP (>6 months) & $148(86.6)$ \\
SC Disability \% (Overall) & $33.3(28.1-38.6)$ \\
SC Disability \% (Low Back) & $5.9(4.1-7.6)$ \\
SC Disability \% (PTSD) & $10.1(6.6-13.6)$ \\
PTSD Diagnosis & $48(28.1)$ \\
Depression Diagnosis & $56(32.8)$ \\
\hline
\end{tabular}

BMI = body mass index, $\mathrm{CI}=$ confidence interval, LBP = low back pain, PTSD = posttraumatic stress disorder, SC = service-connected. 


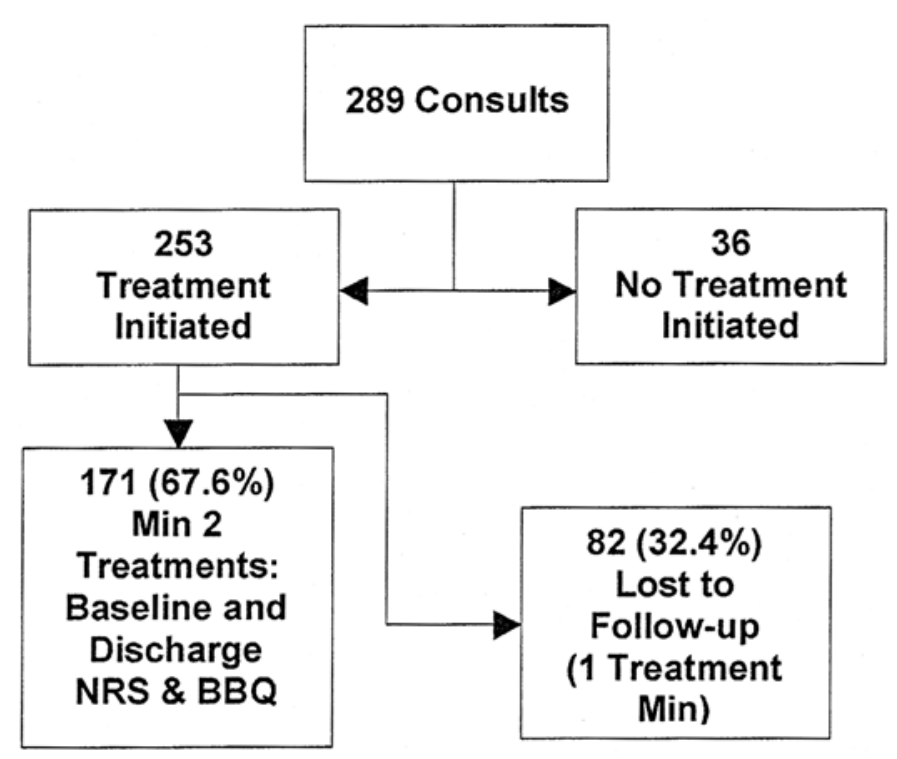

Figure 1.

Clinical course of consultations for low back pain. BBQ = Back Bournemouth Questionnaire, Min $=$ minimum, NRS $=$ Numeric Rating Scale.

8.7 (95\% CI $=8.0-9.3)$, with a range from 2 to 26 . In terms of manual treatment type, alone or in combination, 95.3 percent received FD; 39.8 percent received highvelocity, low amplitude SMT; and 19.3 percent received spinal mobilization without high-velocity thrust.

\section{Clinical Outcomes}

For the NRS, the mean raw score improvement was 2.2 points $(95 \% \mathrm{CI}=1.8-2.5)$ from a baseline score of $5.9(95 \% \mathrm{CI}=5.5-6.2)$ to a discharge score of $3.7(95 \%$ $\mathrm{CI}=3.3-4.1)$, representing a percentage improvement from baseline of 37.4 percent $(95 \% \mathrm{CI}=31.7-43.1)(t=$ 12.37, $p<0.001)$. For the BBQ, the mean raw score improvement was 13.6 points $(95 \% \mathrm{CI}=11.4-15.8)$ from a baseline score of $40.3(95 \% \mathrm{CI}=38.3-42.3)$ to a discharge score of 26.6 (95\% CI = 24.1-29.1), representing a percentage improvement from baseline of 34.6 percent $(95 \%$ CI $=29.3-40.0)(t=12.38, p<0.001)$. Based upon an MCID of 30 percent, 103 patients (60.2\%) met or exceeded that percentage of improvement in terms of NRS and 92 patients (53.8\%) met or exceeded that percentage of improvement in terms of BBQ (Figures 2 and 3, respectively). Mean percentages of clinical improvement were statistically significant and clinically meaningful for both the NRS and BBQ.

\section{DISCUSSION}

The clinical outcomes achieved for this sample should be considered within the context of this veteran patient base, which is typically represented by older, white males with multiple comorbidities. A high percentage of overall service-connected disability was noted, with only a small percentage associated with the low back region. Considerable psychological comorbidity was found, with a high prevalence of PTSD and depression diagnoses. PTSD and chronic pain tend to co-occur and may interact in a way that can negatively affect either disorder [21-23]. A previous retrospective study of chiropractic management for neck and back pain demonstrated less improvement among those with PTSD [11]. These points are significant because severe comorbidities and psychosocial factors lessen the likelihood of obtaining positive outcomes with conservative measures, including SMT, for chronic LBP [6]. Mean percentages of clinical improvement exceeded the MCID, despite the levels of service-connected disability and comorbidity among this sample of veteran patients.

Manual treatment selection was based on the best available evidence in concert with both the clinical judgment of providers and patient preference. The complexity of various patient presentations likely influenced practice patterns within this clinic with regard to manual treatment selection. FD or spinal mobilization may have been used in favor of SMT in the presence of underlying bone weakening disorders (osteoporosis), spondylolysis, or surgical fusion or hardware, where a more gentle approach was deemed appropriate by the provider. While the use of FD for LBP is fairly common among chiropractors, the use of FD on more than 95 percent of patients with LBP far exceeds published accounts outside of the VHA of less than 25 percent [24]. This is contrasted with the use of traditional high-velocity, lowamplitude SMT within this study on just under 40 percent of patients, compared with reports of more than 71 percent outside of the VHA [24]. The recent survey of VHA chiropractors reported that high-velocity, low-amplitude SMT was the most common technique used, suggesting that treatment selection reported in this study differed from chiropractic clinic settings both within and outside the VHA. The reason for this variation is unclear, and additional research among veterans with LBP is warranted to determine the extent to which manual treatment selection influences clinical outcomes. 


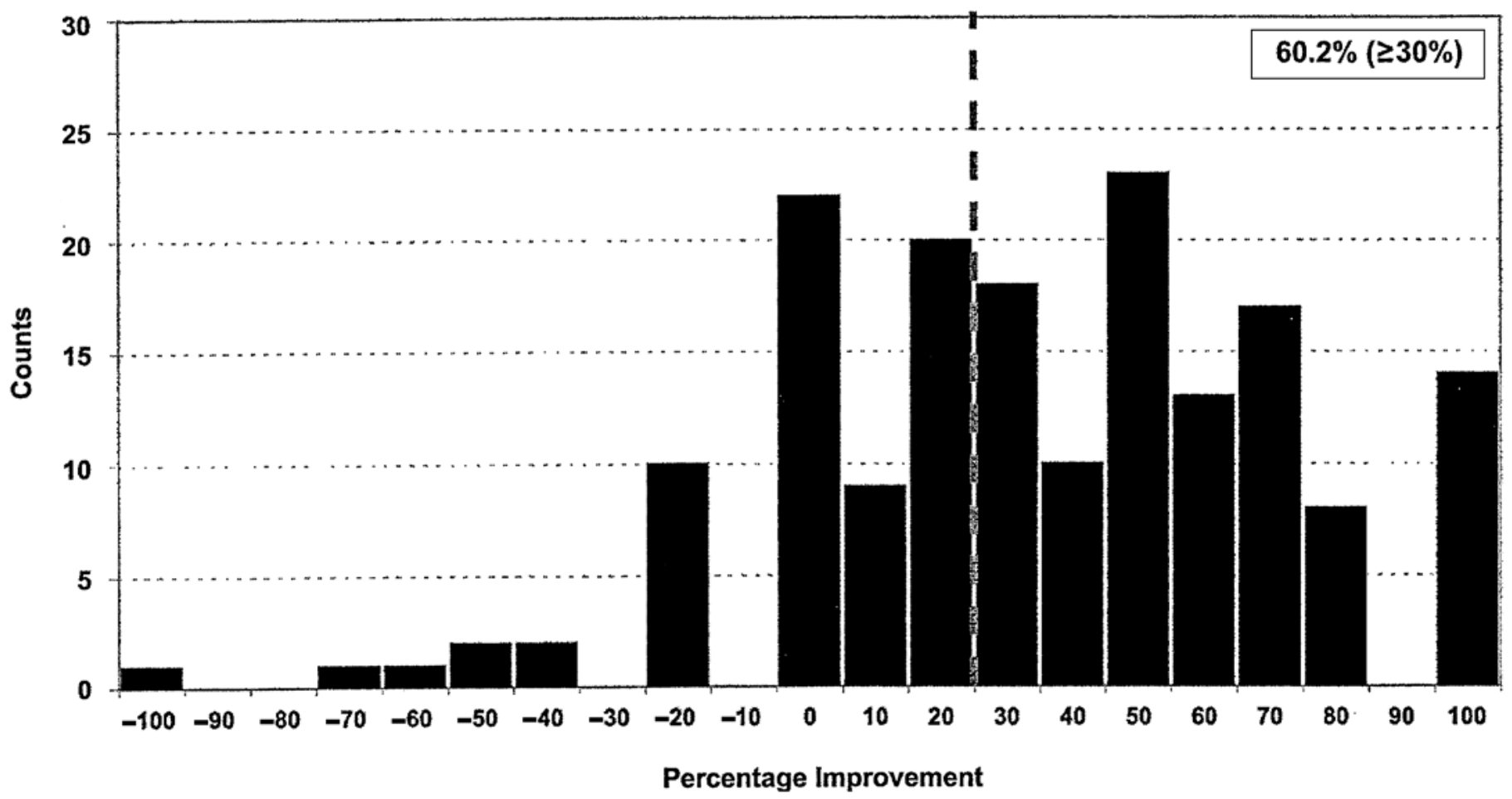

Figure 2.

Histogram of percentage improvement from baseline for Numeric Rating Scale (minimum clinically important difference $\geq 30 \%$ ).

The mean number of treatments was relatively low for this sample at 8.7 visits. This represents the only published account of number of chiropractic treatments for LBP among veteran patients. Unique characteristics of the VHA as an integrated healthcare system limit direct comparisons to chiropractic utilization data outside of the VHA. To provide a context for comparison, we found the number of treatments was similar to the 9.0 chiropractic treatments previously reported for a sample of veterans from varied periods of military service with either neck pain or LBP [11]. If the minimum of four treatments established as inclusion criteria for that previous study was applied to the current study, the mean number of treatments would increase to 9.1, reflective of consistent practice patterns within this clinic setting between 2006 and 2009 [11]. The mean number of treatments within the current study was higher than the 6.1 treatments reported for a smaller OIF/OEF sample with either neck pain or LBP [10]. While the patients treated in this current report achieved a clinically and statistically significant change in LBP over what appears to be a relatively short course of care, future investigations into clinical outcomes and the duration of courses of chiropractic care within the VHA are warranted, with a broader representation of clinic settings and providers.

The outcomes of the present study are different in a number of ways than those observed for LBP management in a previous study by our team [11]. For patients initiating a course of chiropractic care for LBP, the present study had data capture of 67.6 percent (171 of 253) compared with 44.5 percent (130 of 292) from the previous study. Additionally, we replaced the Revised Oswestry Disability Questionnaire [25] (which focuses on self-reported disability) as a primary outcome measure with pain severity (NRS) and a biopsychosocial measure of back pain symptoms (BBQ). In our previous work, clinical outcomes from the Revised Oswestry Disability Questionnaire demonstrated mean percentage improvement from baseline of 17.7 percent (95\% CI 13.2-22.2) [11]. The present study demonstrates clinical improvements of 37.4 percent (NRS) and 34.6 percent (BBQ), without significant differences in the mean number of treatments provided between the two studies [11]. The use of the BBQ may have also contributed to the 


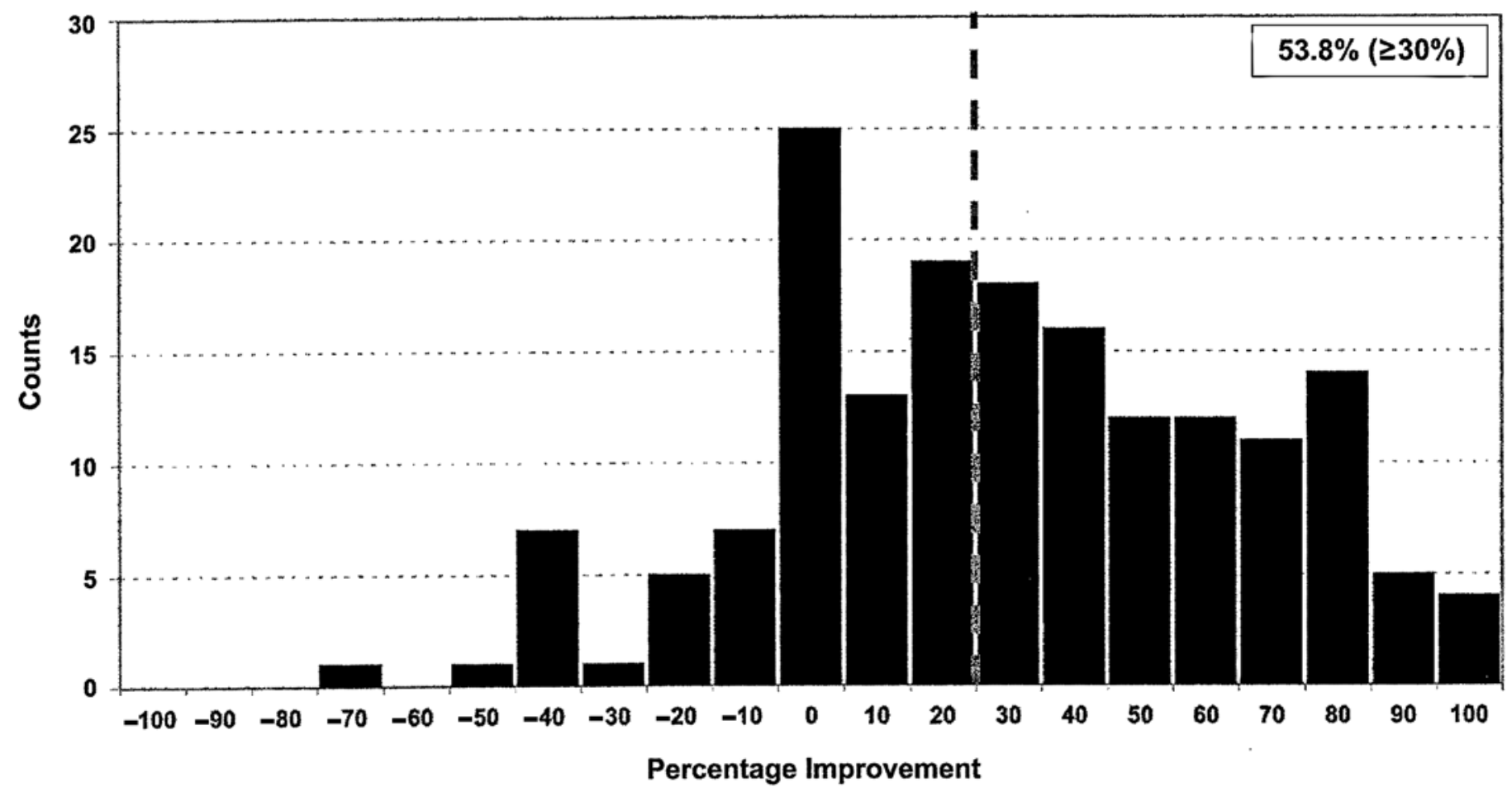

Figure 3.

Histogram of percentage improvement from baseline for Back Bournemouth Questionnaire (minimum clinically important difference $\geq 30 \%$ ).

increased percentage of data capture because it is relatively easy for the patient to complete and the provider to administer. We feel that the use of pain severity (NRS) and a biopsychosocial measure of LBP symptoms (BBQ) are more appropriate measures of treatment response than self-reported disability in a veteran population with a high prevalence of service-connected disability.

While the credibility, accuracy, and comprehensiveness of the data were likely enhanced through the use of a prospectively maintained quality assurance data set, study limitations still include those inherent to the nature of retrospective design. We acknowledge that patients tend to seek care when their pain severity is elevated and thus, on average, tend to experience a reduction in pain severity irrespective of treatment. The effect of this limitation was likely lessened by the predominantly chronic nature of the presenting LBP complaints (86.6\%) and potential delays in access to care associated with the gatekeeper referral process. While treatments were generally provided at a frequency of two times a week, with outcome measures being collected after every four treatments, variations in that frequency and the duration of care could have influenced clinical outcomes. The study design did not control for other variables that may have positively or negatively affected treatment response during the courses of care. While 253 patients with completed consults initiated a course of care, analysis was based upon 171 patients meeting inclusion criteria, with 82 patients being lost to follow-up. The response to treatment is not known for those patients with a minimum of one treatment who were lost to follow-up. Future research with a prospective design could address many of these identified limitations.

\section{CONCLUSIONS}

The increasing prevalence and costs associated with LBP among veterans receiving care within the VHA lend support to investigating veteran responses to conservative forms of management that have demonstrated effectiveness outside of the VHA. This retrospective case series of a prospectively maintained data set advances previous work with improved data capture, increased sample size, and use of NRS and BBQ as outcome measures. Despite high levels of service-connected disability 
and comorbidity, veterans' chiropractic clinical outcomes in terms of mean percentage improvement from baseline to discharge for both NRS and BBQ were statistically significant and clinically meaningful. This study adds to the understanding of chiropractic clinical outcomes for veterans with LBP and contributes to a foundation for further research.

\section{ACKNOWLEDGMENTS}

\section{Author Contributions:}

Study concept and design: A. S. Dunn, B. N. Green.

Acquisition of data: A. S. Dunn, L. R. Formolo.

Analysis and interpretation of data: A. S. Dunn, D. Chicoine.

Drafting manuscript: A. S. Dunn, L. R. Formolo, B. N. Green,

D. Chicoine.

Financial Disclosures: The authors have declared that no competing interests exist.

Funding/Support: This material was based on work supported by and with the use of resources at the VAWNYHS.

Additional Contributions: We thank Carol Simolo of New York Chiropractic College for her valued contributions.

Institutional Review: The study was reviewed and approved through the VAWNYHS Research and Development Committee and Institutional Review Board.

Participant Follow-Up: The authors do not plan to inform participants of the publication of this study.

Disclaimer: The views expressed in this article are those of the authors and do not reflect the official policy or position of the Department of the Navy, Department of Defense, or the U.S. Government.

\section{REFERENCES}

1. Yu W, Ravelo A, Wagner TH, Phibbs CS, Bhandari A, Chen S, Barnett PG. Prevalence and costs of chronic conditions in the VA health care system. Med Care Res Rev. 2003;60(3 Suppl):146S-67S. [PMID: 15095551] DOI:10.1177/1077558703257000

2. Sinnott P, Wagner TH. Low back pain in VA users. Arch Intern Med. 2009;169(14):1338-39. [PMID: 19636039] DOI:10.1001/archinternmed.2009.201

3. Kang HK, Mahan CM, Lee KY, Magee CA, Murphy FM. Illnesses among United States veterans of the Gulf War: A population-based survey of 30,000 veterans. J Occup Environ Med. 2000;42(5):491-501. [PMID: 10824302] DOI:10.1097/00043764-200005000-00006

4. Gironda RJ, Clark ME, Massengale JP, Walker RL. Pain among veterans of Operations Enduring Freedom and Iraqi Freedom. Pain Med. 2006;7(4):339-43. [PMID: 16898945] DOI:10.1111/j.1526-4637.2006.00146.x
5. Dunn AS, Green BN, Gilford S. An analysis of the integration of chiropractic services within the United States military and veterans' health care systems. J Manipulative Physiol Ther. 2009;32(9):749-57. [PMID: 20004802]

DOI:10.1016/j.jmpt.2009.10.009

6. Bronfort G, Haas M, Evans R, Kawchuk G, Dagenais S. Evidence-informed management of chronic low back pain with spinal manipulation and mobilization. Spine J. 2008; 8(1):213-25. [PMID: 18164469$]$

DOI:10.1016/j.spinee.2007.10.023

7. Chou R, Qaseem A, Snow V, Casey D, Cross JT Jr, Shekelle P, Owens DK; Clinical Efficacy Assessment Subcommittee of the American College of Physicians; American College of Physicians; American Pain Society Low Back Pain Guidelines Panel. Diagnosis and treatment of low back pain: A joint clinical practice guideline from the American College of Physicians and the American Pain Society. Ann Intern Med. 2007;147(7):478-91.

[PMID: 17909209]

8. Furlan A, Yazdi F, Tsertsvadze A, Gross A, Van Tulder M, Santaguida L, Cherkin D, Gagnier J, Ammendolia C, Ansari M, Ostermann T, Dryden T, Doucette S, Skidmore B, Daniel R, Tsouros S, Weeks L, Galipeau J. Complementary and alternative therapies for back pain II. Evidence report/technology assessment No. 194. (Prepared by the University of Ottawa Evidence-Based Practice Center under Contract No. 290-2007-10059-I (EPCIII). AHRQ Publication No. 10(11)E007. Rockville (MD): Agency for Healthcare Research and Quality; 2010.

9. Dunn AS, Baylis S, Ryan D. Chiropractic management of mechanical low back pain secondary to multiple-level lumbar spondylolysis with spondylolisthesis in a United States Marine Corps veteran: A case report. J Chiropr Med. 2009; 8(3):125-30. [PMID: 19703668]

DOI:10.1016/j.jcm.2009.04.003

10. Lisi AJ. Management of Operation Iraqi Freedom and Operation Enduring Freedom veterans in a Veterans Health Administration chiropractic clinic: A case series. J Rehabil Res Dev. 2010;47(1):1-6. [PMID: 20437322] DOI:10.1682/JRRD.2009.07.0095

11. Dunn AS, Passmore SR, Burke J, Chicoine D. A crosssectional analysis of clinical outcomes following chiropractic care in veterans with and without post-traumatic stress disorder. Mil Med. 2009;174(6):578-83. [PMID: 19585768]

12. Lisi AJ, Goertz C, Lawrence DJ, Satyanarayana P. Characteristics of Veterans Health Administration chiropractors and chiropractic clinics. J Rehabil Res Dev. 2009;46(8): 997-1002. [PMID: 20157856] DOI:10.1682/JRRD.2009.01.0002 
13. Triano J. The mechanics of spinal manipulation. In: Herzog W, editor. Clinical biomechanics of spinal manipulation. Philadelphia (PA): Churchill Livingstone; 2000. p. 92-190.

14. Downie WW, Leatham PA, Rhind VM, Wright V, Branco JA, Anderson JA. Studies with pain rating scales. Ann Rheum Dis. 1978;37(4):378-81. [PMID: 686873] DOI:10.1136/ard.37.4.378

15. Bolton JE, Breen AC. The Bournemouth Questionnaire: A short-form comprehensive outcome measure. I. Psychometric properties in back pain patients. J Manipulative Physiol Ther. 1999;22(8):503-10. [PMID: 10543579] DOI:10.1016/S0161-4754(99)70001-1

16. Bolton JE, Humphreys BK. The Bournemouth Questionnaire: A short-form comprehensive outcome measure. II. Psychometric properties in neck pain patients. J Manipulative Physiol Ther. 2002;25(3):141-48. [PMID: 11986574] DOI:10.1067/mmt.2002.123333

17. Bolton JE. Sensitivity and specificity of outcome measures in patients with neck pain: Detecting clinically significant improvement. Spine (Phila Pa 1976). 2004;29(21):2410-17. [PMID: 15507803] DOI:10.1097/01.brs.0000143080.74061.25

18. Hurst H, Bolton J. Assessing the clinical significance of change scores recorded on subjective outcome measures. J Manipulative Physiol Ther. 2004;27(1):26-35. [PMID: 14739871] DOI:10.1016/j.jmpt.2003.11.003

19. Ostelo RW, Deyo RA, Stratford P, Waddell G, Croft P, Von Korff M, Bouter LM, De Vet HC. Interpreting change scores for pain and functional status in low back pain: Towards international consensus regarding minimal important change. Spine (Phila Pa 1976). 2008;33(1):90-94. [PMID: 18165753] DOI:10.1097/BRS.0b013e31815e3a10

20. Department of Veterans Affairs. Federal benefits for veterans, dependents, and survivors, 2011 edition [Internet]. Washington (DC): Department of Veterans Affairs; 2011 [updated 2011 Jun 16; cited 2011 Jul 17]. Available from: http://www.va.gov/opa/publications/benefits book.asp.

21. Sharp TJ, Harvey AG. Chronic pain and posttraumatic stress disorder: Mutual maintenance? Clin Psychol Rev.
2001;21(6):857-77. [PMID: 11497210]

DOI:10.1016/S0272-7358(00)00071-4

22. Asmundson GJ, Coons MJ, Taylor S, Katz J. PTSD and the experience of pain: Research and clinical implications of shared vulnerability and mutual maintenance models. Can J Psychiatry. 2002;47(10):930-37. [PMID: 12553128]

23. Otis JD, Keane TM, Kerns RD. An examination of the relationship between chronic pain and post-traumatic stress disorder. J Rehabil Res Dev. 2003;40(5):397-405.

[PMID: 15080224]

DOI:10.1682/JRRD.2003.09.0397

24. Christensen MG; National Board of Chiropractic Examiners. Job analysis of chiropractic 2005: A project report, survey analysis, and summary of the practice of chiropractic within the United States. Greeley (CO): National Board of Chiropractic Examiners; 2005.

25. Hudson-Cook N, Tomes-Nicholson K, Breen AC. A Revised Oswestry Back Disability Questionnaire. In: Roland M, Jenner JR, editors. Back pain: New approaches to education and rehabilitation. New York (NY): Manchester University Press; 1989. p. 197-204.

Submitted for publication October 18, 2010. Accepted in revised form February 28, 2011.

This article and any supplementary material should be cited as follows:

Dunn AS, Green BN, Formolo LR, Chicoine D. Retrospective case series of clinical outcomes associated with chiropractic management for veterans with low back pain. J Rehabil Res Dev. 2011;48(8):927-34.

DOI:10.1682/JRRD.2010.10.0202

ResearcherID: Andrew S. Dunn, DC, MS, MEd: B-59352011.

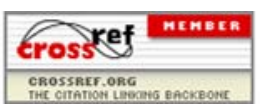

